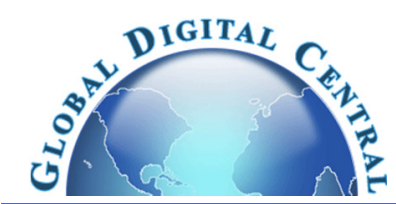

Frontiers in Heat and Mass Transfer

Available at www.ThermalFluidsCentral.org

\title{
IMPACT OF THERMAL RADIATION AND CHEMICAL REACTION ON UNSTEADY 2D FLOW OF MAGNETIC-NANOFLUIDS OVER AN ELONGATED PLATE EMBEDDED WITH FERROUS NANOPARTICLES
}

\author{
S.P. Samrat, C. Sulochana*, G.P. Ashwinkumar \\ Department of Mathematics, Gulbarga University, Gulbarga, Karnataka, 585106, India
}

\begin{abstract}
This study reports the flow, thermal and concentration attributes of magnetic-nanofluids past an elongated plate with thermal radiation and chemical reaction. The flow considered is two-dimensional and time-dependent. The pressure gradient and ohmic heating terms are neglected in this analysis. The flow governing PDEs are transformed into ODEs using appropriate conversions. Further, the set of ODEs are solved analytically using perturbation technique. The flow quantities such as velocity, thermal and concentration fields are discussed under the influence of various pertinent parameters namely volume fraction of nanoparticle, magnetic field, stretching parameter, Soret number, radiation and chemical reaction with the assistance of graphical representations. Moreover, wall friction, reduce Nusselt and Sherwood numbers are calculated and deployed in tabular forms. Dual nature is observed for water-based $\mathrm{Fe}_{3} \mathrm{O}_{4}$ nanofluid and Ethylene glycol based $\mathrm{Fe}_{3} \mathrm{O}_{4}$ nanofluid. The results indicate that water-based ferrofluids are highly influenced as compared with Ethylene Glycol based ferrofluids. Also, the nanoparticle volume fraction plays a vital role in controlling the wall friction and heat transfer performance. Also, Soret parameter has a tendency to integrate high density particles in a boundary to raise the thickness of a surface.
\end{abstract}

Keywords: $M H D$, Nanofluids, Stretching plate, Thermal radiation, Chemical reaction, Soret effect.

\section{INTRODUCTION}

Nanofluid is the deferral of minute particles of the range 1-100 nanometer in various base fluids these nanoparticles are homogeneously and stably dispersed in the pedestal fluids. Nanofluid provide an enhanced thermal conductivity as compared with normal fluids and they are the most innovative and obligatory fluids in many science and engineering applications. Such applications include in power generation, electronic equipment's, Chemical and metallurgical process, air conditioning, medical fields, etc. In view of this, Kim et al. (2004) studied the convective uncertainty and thermal transport nature of nanofluids. Later, Murshed et al. (2005) established the interesting results on enhancing the thermal conductivity of water with $\mathrm{TiO} 2$ nanomaterials. Numerical analysis has been carried out by Oztop and Abu-Nada (2008) to confer the impact of buoyancy forces on the flow and thermal transport nature of nanofluid past a moderately heated rectangular channel. They found that use of heated surface in the flow of nanofluids improved the velocity and thermal fields. The flow of nanofluid over an inclined channel with external heat supply was examined by Öğü (2009), they concluded that increase in the length of heater will decelerate the heat transfer rate for smaller inclination. Further, few researchers [Khan and Pop (2010), Rana and Bhargava (2011), Sarkara et al. (2012), Sher et al. (2013), Trimbitas et al. (2014), Hayat et al. (2014), Reddy et al. (2015), Raju et al. (2016)] explained the flow of nanofluid over dissimilar geometries, such as elongated surface, a vertical plate, thin needle, circular cylinder, flat plate under various boundary conditions. Mahanthesh et al. (2016) elaborated numerically the impact of MHD and thermal slip mechanism on the 3D flow of nanofluid past a linear and nonlinear elongated surface. They provided the nice comparison between linear and nonlinear stretching surface using different nanoparticles with water as the base fluid.

Impact of the inclined magnetic field over the flow of nanofluid caused by a thin liquid film in the presence of graphene nanoparticles was deliberated by Sandeep (2016), the author has produced good results by taking various mixtures of Water, Ethylene glycol with graphene nanoparticles. Babu and Sandeep (2016) investigated the impact of slip mechanism on MHD nanofluid flow past an elongated sheet of variable thickness in the presence of thermophoresis and Brownian motion effects. They produced dual nature by considering $\mathrm{Cu}$ and $\mathrm{CuO}$ nanoparticles in water. They concluded that heat transfer performance of $\mathrm{Cu}$-water nanofluid is more compared with $\mathrm{CuO}$-water nanofluid. The flow of three different nanofluids over an elongated surface in the presence of variable heat source was scrutinized by Sandeep and Sulochana (2016). They found that heat transfer in Jeffrey nanofluid is good as compared with Maxwell and Oldroyd-B nanofluid. Recently, Raza et al. (2016) discussed the flow of nanofluid over a porous channel and Hakeem et al. (2017) provided a comparative analysis of the flow of Newtonian/non-Newtonian nanofluid over a flat surface in the presence of constant heat flux. They concluded that heat transfer performance of non-magnetic nanoparticles is more as compared with magnetic nanoparticles. Very recently, Sulochana et al. [(2017), (2017)] included thermophoresis and Brownian motion mechanisms in their analysis to enhance the thermal transfer phenomenon. Sulochana et al. [(2017), (2017)] reported the nanofluid flow problem by using various combinations of nanofluid ferrous nanoparticles in water, methanol base fluids and silver nanoparticles in water, kerosene base fluids. They found that methanol based ferrofluids possess more thermal transfer rate as compared with water-based ferrofluids and similarly Silver-water 
nanofluid possess more heat transfer rate as compared with Silverkerosene nanofluid.

Flow, heat and mass transfer problems under the impact of chemical reaction, thermal radiation can be found in many scientific and industrial applications. Such areas include chemical industry, petroleum industry, cooling of nuclear reactors, power and cooling industry for drying. The Soret effect is the mass flux created by thermal gradient. It has the number of applications in engineering problems. Impact of cross diffusion and thermal radiation on MHD flow over a porous plate was studied by Vempati and Laxmi-Narayana-Gari (2010) and Hayat et al. (2012). Further, Pal and Mondal (2014) and Krishana et al. (2015) elaborated the chemical reaction, radiation and Soret effects on flow over an elongated surface in the presence of external heat source. Later, several researchers [Srinivasacharya et al. (2015), Sulochana et al. (2016), Reddy and Chamkha (2016), and Sandeep et al. (2015)] discussed the impact of chemical reaction, radiation, heat source Soret effect in their analysis. Further the useful work related to this article can be found in the references such as Rashid et al. (2017), Nadeem and Saleem (2014), Babu et al. (2017), Nadeem et al.(2016), Saleem et al.(2016), Sulochana et al. (2017a,b,c,; 2018).

By making use of aforementioned studies, we filled the gap by extending the work of Sulochana et al. (2017). The motivation of this study is to examine the impact of thermal radiation and thermal diffusion on the flow of chemically reactive nanofluid past a porous elongated sheet in the presence of ferrous nanoparticles. The solutions to the flow governing equations can be derived by using perturbation technique. The impact of various pertinent parameters on velocity, thermal and concentration fields are deployed with help of graphs. For practical interest, we discussed the impact of aforesaid parameters on skin friction factor, local Nusselt number and Sherwood number. Dual nature is observed for water based $\mathrm{Fe}_{3} \mathrm{O}_{4}$ nanofluid and Ethylene Glycol based $\mathrm{Fe}_{3} \mathrm{O}_{4}$ nanofluid.

\section{MATHEMATICAL FORMULATION}

Let us assume, unsteady, incompressible laminar flow natural MHD convective nanofluid flow past a permeable stretching plate. Fig. 1 represents the flow model of the problem. Consider the flow is along $x$ axis with plate and $y$-axis is normal to it. Also the magnetic field $B_{0}(x)$ is applied parallel to $\mathrm{x}$-axis. We assume that an exterior heat source is invariant. It is imagined that the equilibrium states of fluid phase and nanoparticles are similar and moreover there is no applied voltage in the absence of an external magnetic field.

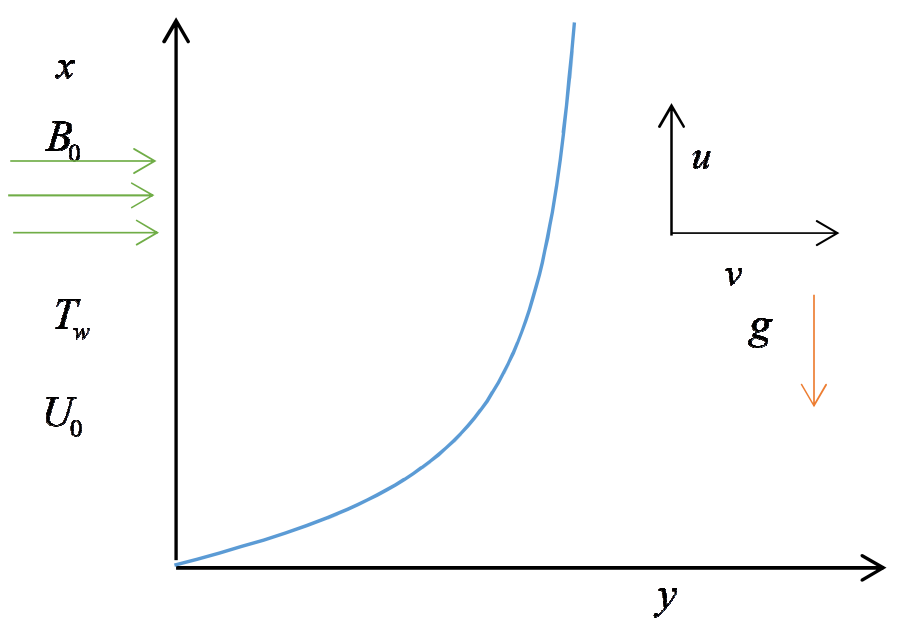

Fig. 1 Flow geometry of the problem

In order to ignore induced magnetic field due to a small Reynolds number is represents an electromagnetic field was effective and associated with the induced magnetic field. Here, $\left(T_{w}, C_{w}\right)$ are wall temperature and concentration of the fluid respectively and $\left(T_{\infty}, C_{\infty}\right)$ are ambient fluid. Soret effect and Thermal radiation effects have been taken into account. The basic governing equations are as follows,

$\frac{\partial \bar{v}}{\partial \bar{y}}=0$

$\rho_{n f}\left(\frac{\partial \bar{u}}{\partial \bar{t}}+v^{*} \frac{\partial \bar{u}}{\partial \bar{y}}\right)=\mu_{n f} \frac{\partial^{2} \bar{u}}{\partial \bar{y}^{2}}-\sigma B_{0}^{2} \bar{u}$,

$\left(\rho C_{p}\right)_{n f}\left(\frac{\partial \bar{T}}{\partial \bar{t}}+v^{*} \frac{\partial \bar{T}}{\partial \bar{y}}\right)=k_{n f} \frac{\partial^{2} \bar{T}}{\partial \bar{y}^{2}}-\frac{16 T_{\infty}^{3} \sigma^{*}}{3 k^{*}} \frac{\partial^{2} \bar{T}}{\partial \bar{y}^{2}}$

$\frac{\partial \bar{C}}{\partial \bar{t}}+\bar{v} \frac{\partial \bar{C}}{\partial \bar{y}}=D_{B} \frac{\partial^{2} \bar{C}}{\partial \bar{y}^{2}}+\frac{D_{m} K_{T}}{T_{m}} \frac{\partial^{2} \overline{\mathrm{T}}}{\partial^{2}}-K_{l}\left(\bar{C}-\bar{C}_{\infty}\right)$,

The corresponding boundary condition is given by,

$\left.\begin{array}{l}\bar{t} \geq 0, \bar{u}(\bar{y}, \bar{t})=0, \bar{T}=\bar{T}_{\infty}, \bar{C}=\bar{C}_{\infty}, \\ \bar{t}<0, \bar{u}(\bar{y}, \bar{t})=U_{0}, \bar{T}=\bar{T}_{w}+\left(\bar{T}_{w}-\bar{T}_{\infty}\right) \varepsilon e^{i w^{\prime} t}, \\ \left.\bar{C}=\bar{C}_{\infty}+\overline{(C}_{w}-\bar{C}_{\infty}\right) \varepsilon e^{i w^{\prime} t} \text { at } \bar{y}=0, \\ \bar{u}(\bar{y}, \bar{t})=0, \bar{T}=\bar{T}_{\infty}, \bar{C}=\bar{C}_{\infty} \text { as } \bar{y} \rightarrow \infty\end{array}\right\}$

where $\bar{u}$ and $\bar{v}$ are velocity components of $x$ and $y$-direction respectively. $\mu_{n f}$ is the viscosity of nanofluid, $\rho_{n f}$ is the density of nanofluid, $\mu_{f}$ is the viscosity of a base fluid, $\sigma$ is the electrical conductivity of a base fluid, and $\left(\rho C_{p}\right)_{n f}$ is the specific heat capacitance

$$
\left.\left.\begin{array}{l}
\rho_{n f}=(1-\phi) \rho_{f}+\phi \rho_{s}, \\
\left(\rho C_{p}\right)_{n f}=(1-\phi)\left(\rho C_{p}\right)_{f}+\phi\left(\rho C_{p}\right)_{s}, \\
(\rho \beta)_{n f}=(1-\phi)(\rho \beta)_{f}+\phi(\rho \beta)_{s},
\end{array}\right\}, \begin{array}{l}
k_{n f}=k_{f}\left(\frac{k_{s}+2 k_{f}-2 \phi\left(k_{f}-k_{s}\right)}{k_{s}+2 k_{f}+2 \phi\left(k_{f}-k_{s}\right)}\right), \\
\alpha_{n f}=\frac{k_{n f}}{\left(\rho C_{p}\right)_{n f}}, \mu_{n f}=\frac{\mu_{f}}{(1-\phi)^{2.5}}, \\
\bar{v}=-V_{0},
\end{array}\right\}
$$

Table 1 Thermophysical properties of nanoparticle and base fluids Sheikholeslami, M., Rokni, H.B., (2017).

\begin{tabular}{|c|c|c|c|}
\hline Physical properties & Ethylene glycol & Water & $\mathrm{Fe}_{3} \mathrm{O}_{4}$ \\
\hline$C_{p}(\mathrm{~J} / \mathrm{kgK})$ & 2400 & 4179 & 670 \\
\hline$\rho\left(\mathrm{kg} / \mathrm{m}^{3}\right)$ & 1110 & 997.3 & 5200 \\
\hline$K(\mathrm{w} / \mathrm{m} K)$ & 0.26 & 0.613 & 6 \\
\hline$\sigma(s / m)$ & $3.41 \times 10^{-3}$ & 21 & $0.74 \times 10^{6}$ \\
\hline
\end{tabular}


where $-V_{0}$ is constant which represents normal in suction and injection case ( $V_{0}>0$ and $V_{0}<0$ resp.). $\phi$ is the volume fraction of a nanoparticle. To develop specific analysis of governing equations we introduce following similarity variable as shown below:

$$
\left.\begin{array}{l}
u=\frac{\bar{u}}{U_{0}}, y=\frac{U_{0} \bar{y}}{v_{f}}, t=\frac{U_{0}^{2} \bar{t}}{v_{f}}, \\
\theta=\frac{\left(\bar{T}-\bar{T}_{\infty}\right)}{\left(\bar{T}_{w}-\bar{T}_{\infty}\right)}, \psi=\frac{\left(\bar{C}-\bar{C}_{\infty}\right)}{\left(\bar{C}_{w}-\bar{C}_{\infty}\right)},
\end{array}\right\}
$$

by using Eqn. (9) the governing Eqn. (2) to (4) are reduced in following form,

$$
\begin{aligned}
& A\left(u_{t}-S u_{y}\right)=D u_{y y}-M u, \\
& \operatorname{Pr} C\left(\theta_{t}-S \theta_{y}\right)=(E+F) \theta_{y y}, \\
& \left(\psi_{t}-S \psi_{y}\right)=(1 / S c) \psi_{y y}+S r \theta_{y y}-K r \psi,
\end{aligned}
$$

with corresponding boundary conditions as

$t<0: u=0, \theta=0, \psi=0$,

$t \geq 0: \mathrm{u}=1, \theta=1+\varepsilon e^{i w t}, \psi=1+\varepsilon e^{i w t}$ at $y=0$,

$u=0, \theta=0, \psi=0$ as $y \rightarrow \infty$,

where $\phi$ is volume fraction, $\operatorname{Pr}$ is Prandtl number, $R$ is radiation parameter, $S r$ is Soret number, $S$ is stretching parameter, $S c$ is Schmidt number, $M$ is magnetic parameter, $K r$ is chemical reaction are as follows,

$$
\left.\begin{array}{l}
\operatorname{Pr}=K_{f} /\left(\mu C_{p}\right)_{f}, R=16 T_{\infty}^{3} \sigma^{*} / 3 K_{f} k^{*}, \\
S=V_{0} / U_{0}, S r=D_{B} K_{T}\left(C^{\prime}-C_{\infty}^{\prime}\right) / T_{m} v_{f}\left(T_{w}^{\prime}-T_{\infty}^{\prime}\right), \\
S c=v_{f} / D_{B}, M=\sigma B_{0}^{2} v_{f} / \rho_{f} U_{0}^{2}, K r=K_{l} v_{f} / U_{0}^{2}
\end{array}\right\}
$$

\section{SOLUTION OF THE PROBLEM}

The coupled nonlinear partial differential equations (10) to (12) with corresponding boundary condition (13) are solved by using a simple perturbation technique. The perturbed expressions are shown below:

$$
\begin{aligned}
& u(y, t)=f_{0}+\varepsilon f_{1} e^{i w t}+o\left(\varepsilon^{2}\right), \\
& \theta(y, t)=g_{0}+\varepsilon g_{1} e^{i w t}+o\left(\varepsilon^{2}\right), \\
& \psi(y, t)=h_{0}+\varepsilon h_{1} e^{i w t}+o\left(\varepsilon^{2}\right),
\end{aligned}
$$

By substituting Eqn. (14) to (16) into Eqn. (10)-(12) and equating coefficient of a like power of $\varepsilon$ and omitting higher terms $\mathcal{E}^{2}$, we obtain the following equations

Zero-order terms:

$D f_{0}^{\prime \prime}+A S f_{0}^{\prime}-M f_{0}=0$,

$(E+R) g_{0}^{\prime \prime}+\operatorname{Pr} C S g_{0}^{\prime}=0$,

$h_{0}^{\prime \prime}+S S c h_{0}^{\prime}-K_{r S c h}=-S r S c g_{0}^{\prime \prime}$,

First order terms:

$D f_{1}^{\prime \prime}+A S f_{1}^{\prime}-(M+A i w) f_{1}=0$,

$(E+R) g_{1}^{\prime \prime}+\operatorname{Pr} C S g_{1}^{\prime}-\operatorname{Pr} C i w g_{1}=0$,

$h_{1}^{\prime \prime}+S S c h_{1}^{\prime}-S c(K r+i w) h_{1}=-S r S c g_{1}^{\prime \prime}$,

the corresponding boundary conditions are

$u_{0}=1, u_{1}=0, \theta_{0}=1, \theta_{1}=1, \psi_{0}=1, \psi_{1}=1$ at $y=0$,

$u_{0}=0, u_{1}=0, \theta_{0}=0, \theta_{1}=0, \psi_{0}=0, \psi_{1}=0$ as $y \rightarrow \infty$, solving the Eqn. (17) to (22) with boundary condition (23), the obtained results as follows:

$$
\begin{aligned}
& u(y, t)=e^{-A_{5} y}+\varepsilon e^{-A_{6} y} e^{i w t}, \\
& \theta(y, t)=e^{-A_{1} y}+\varepsilon\left(e^{-A_{2} y}\right) e^{i w t} \text {, } \\
& \left.\begin{array}{rl}
\psi(y, t)= & \left(\left(1-j_{1}\right) e^{-A_{3} y}-j_{1} e^{-A_{1} y}\right)+ \\
& \varepsilon\left(\left(1-j_{2}\right) e^{-A_{4} y}-j_{2} e^{-A_{2} y}\right) e^{i w t},
\end{array}\right\}
\end{aligned}
$$

The friction factor, local Nusselt number and Sherwood number are defined as follows:

$$
\begin{aligned}
& C_{f}=\left.\frac{\partial u}{\partial t}\right|_{y=0}=A_{5}-\varepsilon A_{6} e^{i w t}, \\
& N u=\left.\frac{\partial \theta}{\partial t}\right|_{y=0}=A_{1}+\varepsilon A_{2} e^{i w t}, \\
& S h=\left.\frac{\partial \psi}{\partial t}\right|_{y=0}=\left(\left(1-j_{1}\right) A_{3}-j_{1} A_{1}\right)+\varepsilon\left(\left(1-j_{2}\right) A_{4}-j_{2} A_{2}\right) e^{i w t},
\end{aligned}
$$

\section{RESULTS AND DISCUSSION}

The structures of nonlinear ODEs (10) to (12) with consistent boundary limits (13) are resolved systematically by using simple perturbation process. Results spell the impact of dimensionless parameter like magnetic field, Prandtl number, stretching parameter, thermal radiation, Soret parameter, volume fraction, and chemical reaction versus momentum, thermal and solute concentration contours along with flow, heat and mass rate are presented by exhausting graphs and tables.

The influence of magnetic field parameter $\mathrm{M}$ on velocity profile is demonstrated in Fig. 2. It is observed that velocity profile is escalated with growing magnetic parameter. The magnetic effect amplifies resistive forces contrasting to the flow which commonly identified as Lorentz force, due to a reason we have seen lessening in flow profile. Fig. 3 has shown the bearing of thermal radiation over a temperature profile. It clears that floating the values of thermal radiation enhances temperature profile. Basically, thermal radiation encompasses an external heat energy which encapsulates movements of conductive particle leads to rise in thermal profile. But reverse action have seen in Fig.4 for various impacts of Prandtl number over a thermal profile. Prandtl number is a proportion of kinematic viscosity to thermal diffusivity so that a growing value of Prandtl number declines the thermal diffusivity.

Variation of volume fraction over velocity, thermal and concentration profile is shown in Figs. 5 to 7 respectively. It is noticed that velocity of the flow diminishes by developing impact of volume fraction, but an opposite effecting have seen in both thermal and concentration field. Increasing the volumetric size or shape of the nanoparticle enhances the density and concentration of nanofluid which leads to enrich thermal and concentration profile but it condenses the flow velocity of a nanofluid. Deviation of stretching parameter over a velocity, thermal and concentration profile has displayed in Figs. 8 to 10 respectively. It clears that rise in stretching parameter shrinks the velocity, thermal and concentration profile. The boundary layer turns into thinner by augmenting stretching parameter, so that we have seen diminishing profiles.

Table 1 displays the thermo-physical properties of nanoparticle and the base fluids. Table 2 and 3 presents the friction factor, local Nusselt and Sherwood number for various impacts of physical parameter with $\mathrm{Fe}_{3} \mathrm{O}_{4}$ nanoparticle suspended in water and ethylene glycol. It is noticed that increasing Prandtl number increases heat transfer rate and simultaneously declines mass transfer rate. Flow rate is increases by increasing magnetic and stretching parameter. Sherwood number is enhances by floating Soret and Chemical reaction parameter. 


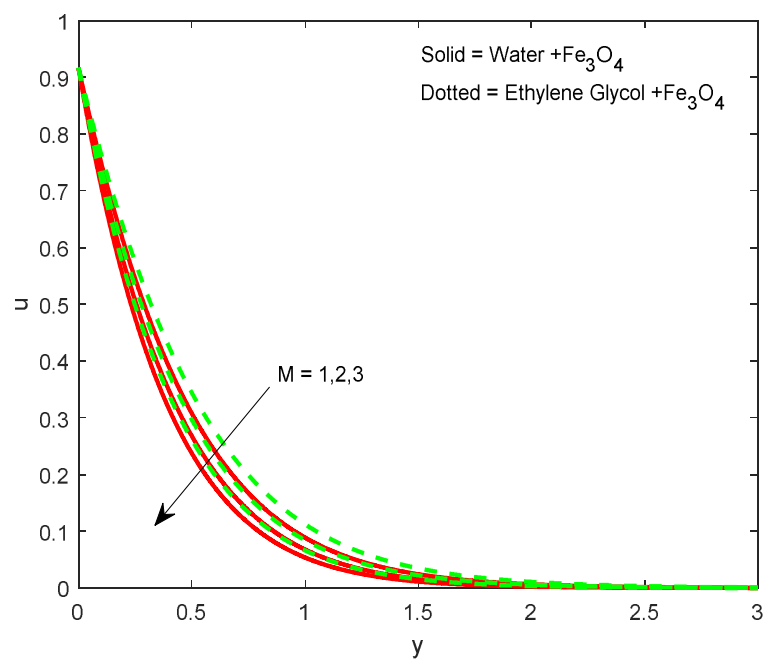

Fig. 2 Impact of $M$ of velocity field.

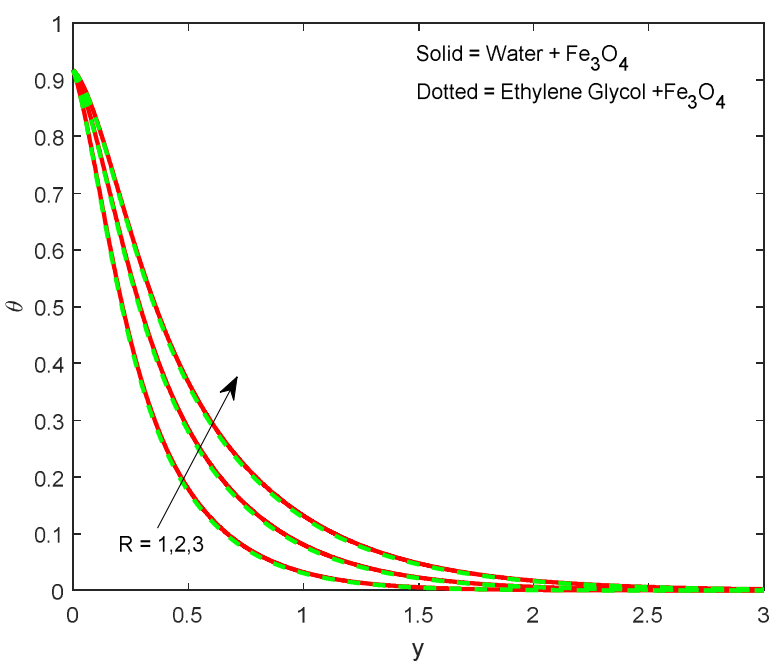

Fig. 3 Impact of $R$ on thermal field.

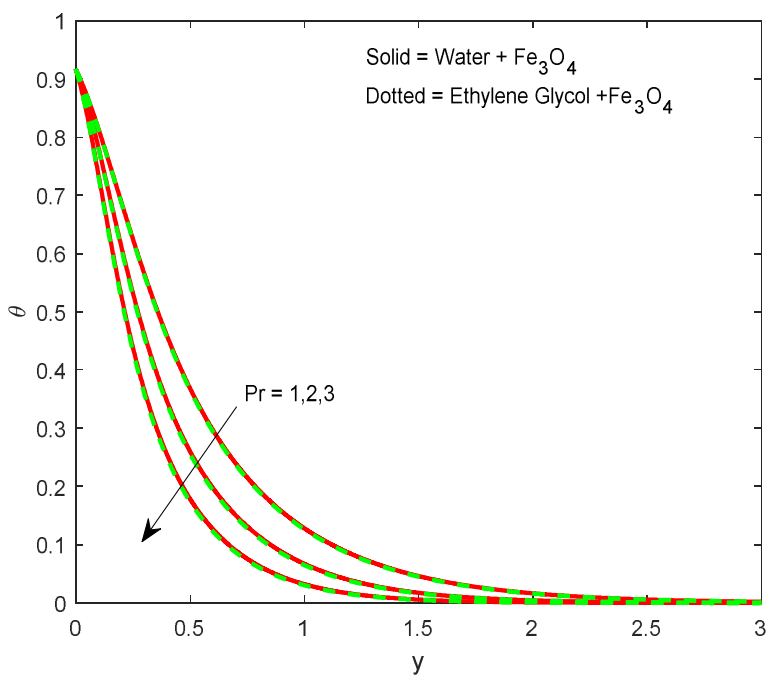

Fig. 4 Impact of Pr on thermal field.

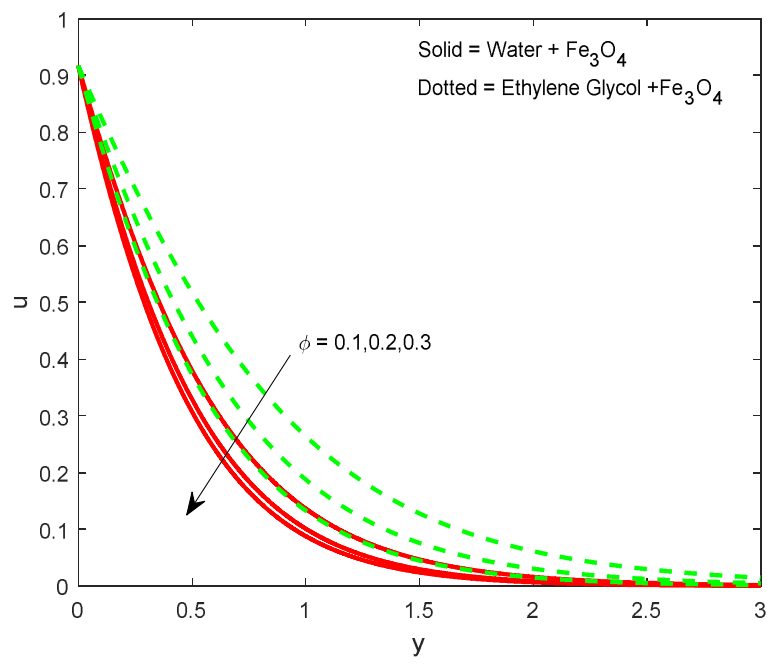

Fig. 5 Impact of $\phi$ on velocity field.

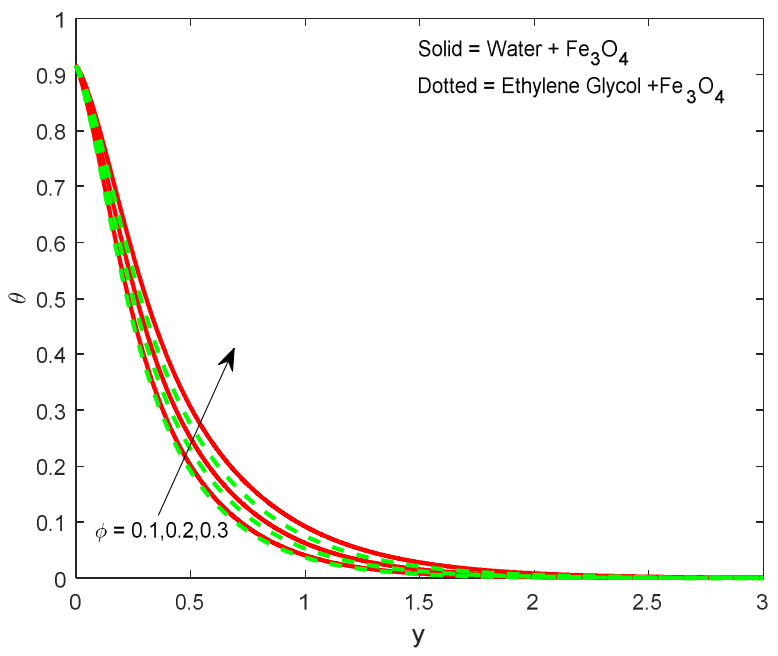

Fig. 6 Impact of $\phi$ on thermal field.

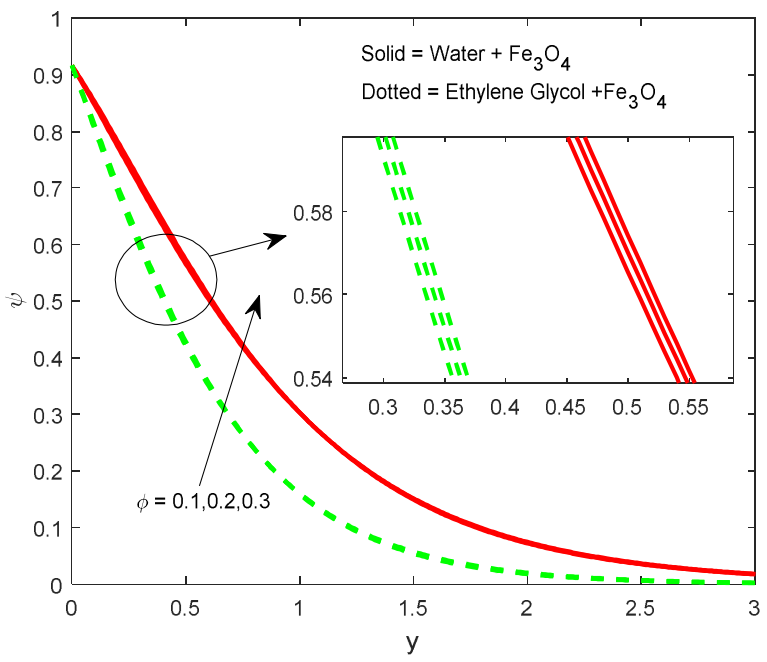

Fig. 7 Impact of $\phi$ on Concentration field. 


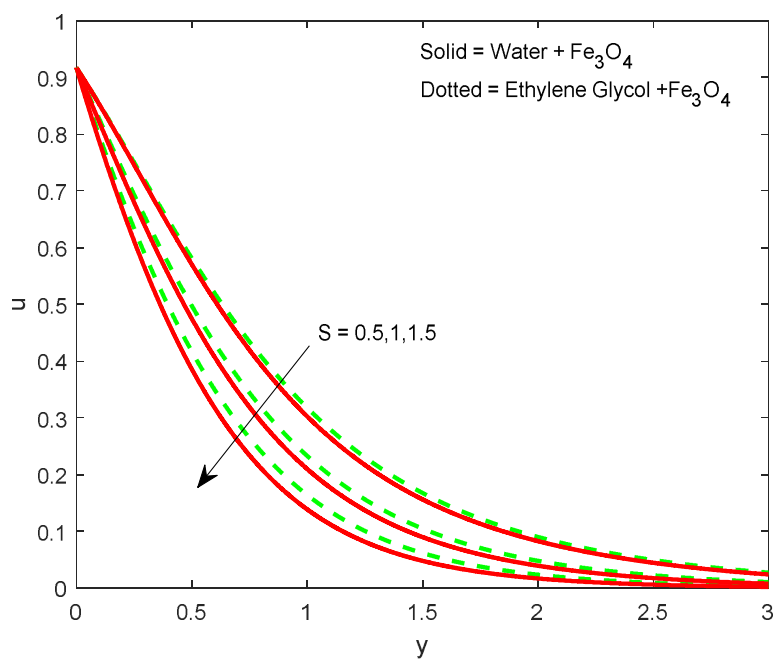

Fig. 8 Impact of $S$ on velocity field.

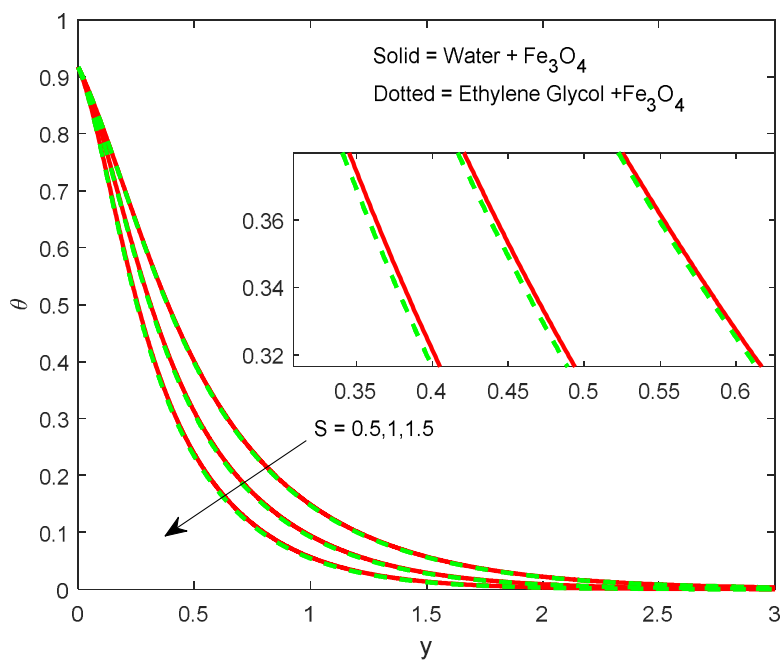

Fig. 9 Impact of $S$ on thermal field.

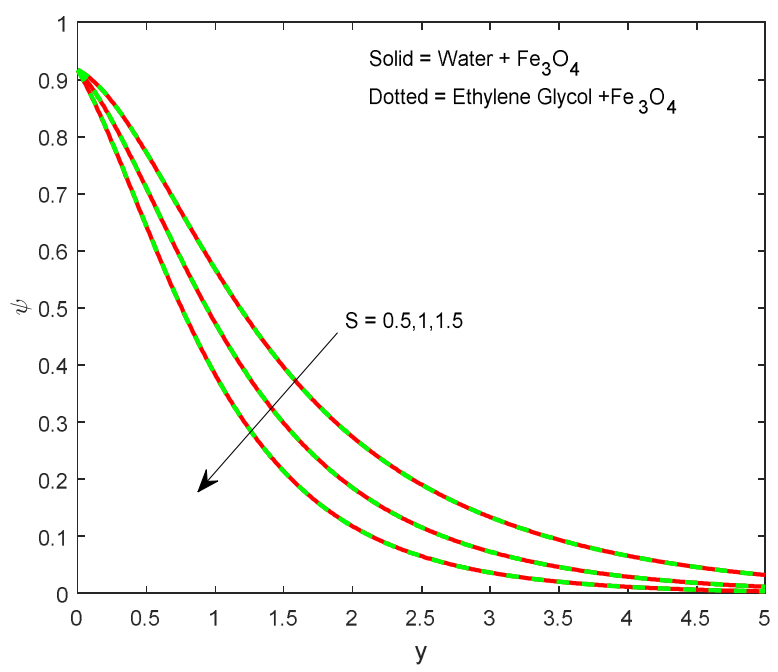

Fig. 10 Impact of $S$ on concentration field.

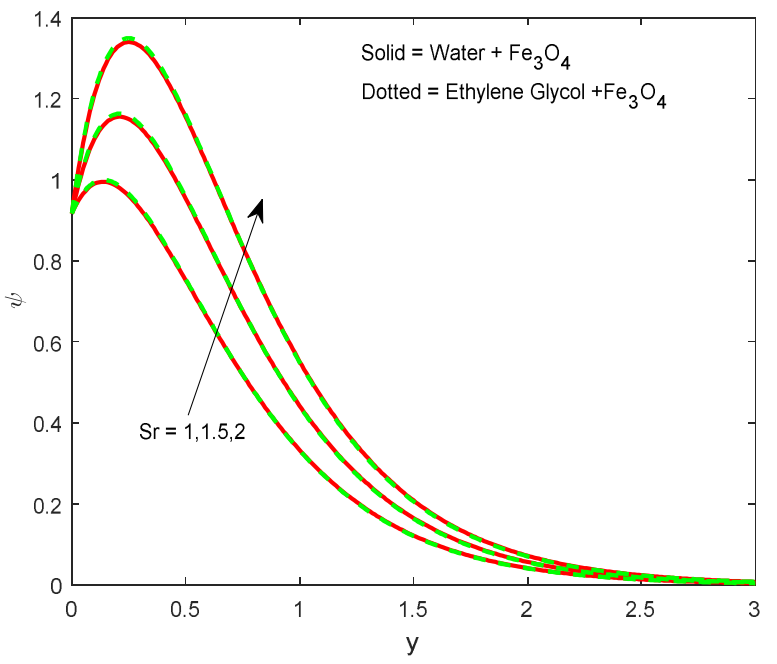

Fig. 11 Impact of $\mathrm{Sr}$ on concentration field.

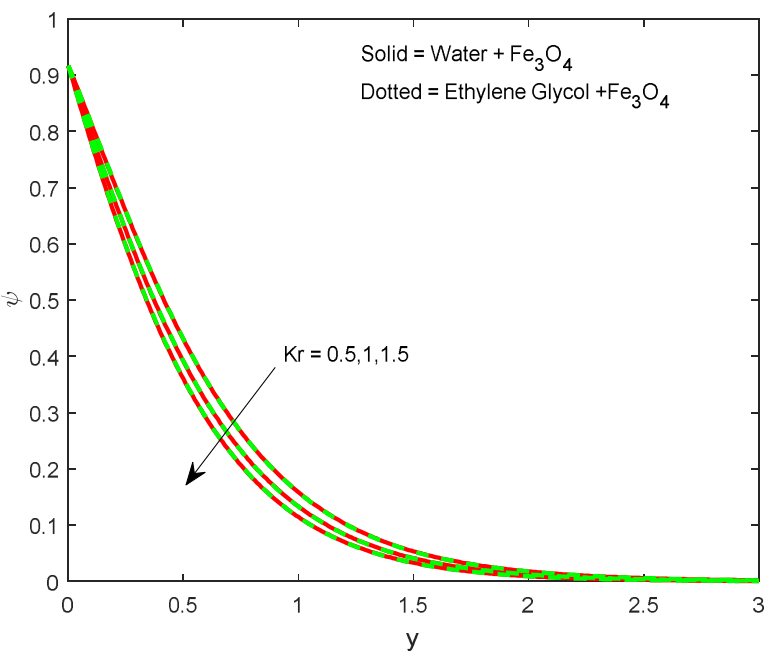

Fig. 12 Impact of $K r$ on concentration field.

Effect of Soret parameter over a concentration profile is displayed in Fig. 11. It is noticed that increment of Soret parameter develops concentration of the flow. The Soret effect is a mixture of particles in liquid and it is highly depends on density and concentration of colloidal particles. It integrates high density particles in a boundary to raise the thickness of a surface causes to upsurge concentration of the flow. An effect of homogeneous chemical reaction over a concentration profile has shown in Fig. 12. It is observed that concentration profile decreases by increasing chemical parameter. Chemical reaction parameter has a tendency to reduce the molecular diffusivity which can cause to decline the thickness of the surface. So that concentration gradient declines by reducing surface thickness.

Table 3 is drawn to validate the obtained analytical results with the various numerical techniques for the effect of magnetic field parameter on the skin friction factor. Table 4 is displayed to validate the obtained results with the previously published literature of Sulochana and Samrat (2017) for limiting cases. 
Table 2 Variation of $\mathrm{C}_{f}, N u_{x}$ and $\mathrm{Sh}_{x}$ for $\mathrm{Fe}_{3} \mathrm{O}_{4}$-Water nanofluid.

\begin{tabular}{|l|l|l|l|l|l|l|l|l|l|}
\hline$\phi$ & $M$ & $R$ & $S$ & $S r$ & $K r$ & $\operatorname{Pr}$ & $C_{f}$ & $N u_{x}$ & $S h_{x}$ \\
\hline 0.1 & & & & & & & 1.9952 & 2.5932 & 0.6564 \\
\hline 0.2 & & & & & & & 1.8402 & 2.2069 & 0.7026 \\
\hline 0.3 & & & & & & & 1.5661 & 1.8616 & 0.7441 \\
\hline & 1 & & & & & & 2.0132 & 2.8038 & 0.6313 \\
\hline & 2 & & & & & & 2.2771 & 2.8038 & 0.6313 \\
\hline & 3 & & & & & & 2.5087 & 2.8038 & 0.6313 \\
\hline & & 1 & & & & & 2.0132 & 2.8038 & 0.6313 \\
\hline & & 2 & & & & & 2.0132 & 1.9882 & 0.7288 \\
\hline & & 3 & & & & & 2.0132 & 1.5632 & 0.7801 \\
\hline & & & 0.5 & & & & 0.8836 & 1.2543 & 0.2273 \\
\hline & & & 1 & & & & 1.2156 & 1.7114 & 0.3311 \\
\hline & & & 1.5 & & & & 1.5961 & 2.2349 & 0.4701 \\
\hline & & & & 1 & & & 2.0132 & 2.8038 & 0.7754 \\
\hline & & & & 1.5 & & & 2.0132 & 2.8038 & 1.6135 \\
\hline & & & & 2 & & & 2.0132 & 2.8038 & 2.4594 \\
\hline & & & & & 0.5 & & 2.0132 & 2.8038 & 0.6313 \\
\hline & & & & & 1 & & 2.0132 & 2.8038 & 0.8105 \\
\hline & & & & & 1.5 & & 2.0132 & 2.8038 & 0.9658 \\
\hline & & & & & & 1 & 2.0132 & 0.915 & 0.8523 \\
\hline & & & & & & 2 & 2.0132 & 1.2328 & 0.8152 \\
\hline & & & & & & 3 & 2.0132 & 1.5954 & 0.7727 \\
\hline
\end{tabular}

Table 3 Variation of $\mathrm{C}_{f}, N u_{x}$ and $\mathrm{Sh}_{x}$ for $\mathrm{Fe}_{3} \mathrm{O}_{4}$ - Ethylene glycol

\begin{tabular}{|l|l|l|l|l|l|l|l|l|l|}
\hline$\phi$ & $M$ & $R$ & $S$ & $S r$ & $K r$ & $\operatorname{Pr}$ & $C_{f}$ & $N u_{x}$ & $S h_{x}$ \\
\hline 0.1 & & & & & & & 1.6851 & 2.6941 & 0.6443 \\
\hline 0.2 & & & & & & & 1.3786 & 2.3758 & 0.6822 \\
\hline 0.3 & & & & & & & 1.0819 & 2.0704 & 0.7188 \\
\hline & 1 & & & & & & 1.8373 & 2.8586 & 0.6248 \\
\hline & 2 & & & & & & 2.1145 & 2.8586 & 0.6248 \\
\hline & 3 & & & & & & 2.3549 & 2.8586 & 0.6248 \\
\hline & & 1 & & & & & 1.8373 & 2.8586 & 0.6248 \\
\hline & & 2 & & & & & 1.8373 & 2.8586 & 0.6248 \\
\hline & & 3 & & & & & 1.8373 & 2.8586 & 0.6248 \\
\hline & & & 0.5 & & & & 0.8795 & 1.2656 & 0.2264 \\
\hline & & & 1 & & & & 1.1623 & 1.7356 & 0.3285 \\
\hline & & & 1.5 & & & & 1.4843 & 2.2740 & 0.4655 \\
\hline & & & & 1 & & & 1.8373 & 2.8586 & 0.8079 \\
\hline & & & & 1.5 & & & 1.8373 & 2.8586 & 1.6636 \\
\hline & & & & 2 & & & 1.8373 & 2.8586 & 2.5265 \\
\hline & & & & & 0.5 & & 1.8373 & 2.8586 & 0.6248 \\
\hline & & & & & 1 & & 1.8373 & 2.8586 & 0.8039 \\
\hline & & & & & 1.5 & & 1.8373 & 2.8586 & 0.9592 \\
\hline & & & & & & 1 & 1.8373 & 1.2449 & 0.8136 \\
\hline & & & & & & 2 & 1.8373 & 2.0166 & 0.7233 \\
\hline & & & & & & 3 & 1.8373 & 2.8586 & 0.6248 \\
\hline
\end{tabular}

\section{CONCLUSIONS}

Present study investigates the impact of thermal radiation and chemical reaction on the flow of magnetic-nanofluids past an elongated sheet in the presence of ferrous nanoparticles. The influence of several physical parameters on velocity, thermal and concentration fields are discussed with help of graphs. The inference of a work is as follows,
- Influence of thermal radiation can holds the stability velocity and thermal fields.

- Soret parameter has a tendency to integrate high density particles in a boundary to raise the thickness of a surface.

- Increasing the wideness of boundary surface roots to decay velocity, thermal and concentration of the flow.

- Nanoparticle size or shape has a propensity to integrate the heat transfer.

- $\mathrm{Fe}_{3} \mathrm{O}_{4}$-water based nanofluid provides more effective heat transfer compare to $\mathrm{Fe}_{3} \mathrm{O}_{4}$-Ethyline glycol nanofluid.

Table 3 Validation of the analytical results with the numerical techniques for skin friction co efficient $C_{f}$

\begin{tabular}{|c|c|c|c|}
\hline $\mathrm{M}$ & $\begin{array}{c}\text { Perturbation } \\
\text { (Present } \\
\text { results) }\end{array}$ & $\begin{array}{c}\text { Shooting } \\
\text { method }\end{array}$ & Bvp5c \\
\hline 1 & 1.8373 & 1.837365514 & 1.837365512 \\
\hline 2 & 2.1145 & 2.114535341 & 2.114535340 \\
\hline 3 & 2.3549 & 2.354981421 & 2.354981421 \\
\hline
\end{tabular}

Table 4 Validation of current study with prescribed results where $R=K r=S c=0, K=\infty$

\begin{tabular}{|c|c|c|}
\hline & $\begin{array}{c}\text { Sulochana and } \\
\text { Samrat (2017) }\end{array}$ & Present study \\
\hline S & Skin friction & Skin friction \\
\hline 1.0 & 1.2881 & 1.2913 \\
\hline 1.5 & 1.8440 & 1.8431 \\
\hline
\end{tabular}

\section{ACKNOWLEDGEMENT}

The third author acknowledge the UGC for financial support under the UGC National Fellowship for Scheduled Caste Students (NFSC) Scheme (No.F1-17.1/2017-18/RGNF-2017-18-SC-KAR-36100).

\section{REFERENCES}

Kim, J., Kang, Y. T., and Choi, C. K., 2004, "Analysis of Convective Instability and Heat Transfer Characteristics of Nanofluids," Phys. Fluids 16, 2395-2401. http://dx.doi.org/10.1063/1.1739247

Murshed, S.M.S., Leong, K.C., Yang, C., 2005, "Enhanced Thermal Conductivity of $\mathrm{TiO}_{2}$-water Based Nanofluids," Int. J. Therm. Sci. 44 367-373. http://dx.doi.org/10.1016/j.ijthermalsci.2001.12.005

Oztop, H. F., and Abu-nada, E., 2008, "Numerical Study of Natural Convection in Partially Heated Rectangular Enclosures Filled with Nanofluids," Int. J. Heat Fluid Flow 29, 1326-1336. http://dx.doi.org/10.1016/j.ijheatfluidflow.2008.04.009

Ogut, E. B., 2009, "Natural Convection of Water-Based Nanofluids in an Inclined Enclosure with a Heat Source," Int. J. Therm. Sci. 48, 2063 2073. http://dx.doi.org/10.1016/j.ijthermalsci.2009.03.014

Khan, W. A., and Pop, I., 2010, "Boundary-Layer Flow of a Nanofluid Past a Stretching Sheet," Int. J. Heat Mass Transf. 53, 2477-2483. http://dx.doi.org/10.1016/j.ijheatmasstransfer.2010.01.032

Rana, P., and Bhargava, R., 2011, "Numerical Study of Heat Transfer Enhancement in Mixed Convection Flow along a Vertical Plate with 
Heat Source/Sink utilizing nanofluids," Commun. Nonlinear Sci. Numer. Simul. 16, 4318-4334. http://dx.doi.org/10.1016/i.cnsns.2011.03.014

Sarkar, S., Ganguly, S., and Biswas, G., 2012, "Mixed Convective Heat Transfer of Nanofluids Past a Circular Cylinder in Cross Flow in Unsteady Regime," Int. J. Heat Mass Transf. 55, 4783-4799. http://dx.doi.org/10.1016/j.ijheatmasstransfer.2012.04.046

Sher, N., Nadeem, S., and Haq, R. U., 2013, "Radiation Effects on MHD Stagnation Point flow of Nano Fluid Towards a Stretching Surface With Convective Boundary Condition," Chinese J. Aeronaut. 26, 1389-1397. http://dx.doi.org/10.1016/j.cja.2013.10.008

Trimbitas, R., Grosan, T., and Pop, I., 2014, "Mixed Convection Boundary Layer Flow along Vertical Thin Needles in Nanofluids," Int. J. Numer. Methods Heat Fluid Flow 24, 579-594. http://dx.doi.org/10.1108/HFF-05-2012-0098

Hayat, T., Imtiaz, M., Alsaedi, A., and Mansoor, R., 2014, "MHD Flow of Nanofluids over an Exponentially Stretching Sheet in a Porous Medium with Convective Boundary Conditions," Chin. Phys. B 23, 1-8. http://dx.doi.org/10.1088/1674-1056/23/5/054701

Reddy, J. V. R., Sugunamma, V., Sandeep, N., and Sulochana, C., 2015, "Influence of Chemical Reaction, Radiation and Rotation on MHD Nanofluid Flow Past a Permeable Flat Plate in Porous Medium," J. Niger. Math. Soc. 35, 48-65. http://dx.doi.org/10.1016/j.jnnms.2015.08.004

Raju, C. S. K., Sandeep, N., and Malvandi, A., 2016, "Free Convective Heat and Mass Transfer of MHD Non-Newtonian Nanofluids over a Cone in the Presence of Non-uniform Heat Source/Sink," J. Mol. Liq. 221, 108-115. http://dx.doi.org/10.1016/j.molliq.2016.05.078

Mahanthesh, B., Gireesha, B. J., Gorla, R. S. R., and Makinde, O. D., 2016, "Magnetohydrodynamic Three-Dimensional Flow of Nanofluids with Slip and Thermal Radiation over a Nonlinear Stretching Sheet: a Numerical Study," Neural Comput. Appl., 1-11. http://dx.doi.org/10.1007/s00521-016-2742-5

Sandeep, N., 2016, "Effect of Aligned Magnetic Field on Liquid Thin Film Flow of Magnetic-Nanofluids Embedded with Graphene Nanoparticles," Adv. Powder Technol. 28, 865-875. http://dx.doi.org/10.1016/j.apt.2016.12.012

Babu, M. J., and Sandeep, N., 2016, "Three-Dimensional MHD Slip Flow of Nanofluids over a Slendering Stretching Sheet with Thermophoresis and Brownian Motion Effects," Adv. Powder Technol. http://dx.doi.org/10.1016/j.apt.2016.07.013

Sandeep, N., and Sulochana, C., 2016, "Momentum and Heat Transfer Behaviour of Jeffrey, Maxwell and Oldroyd-B Nanofluids Past a Stretching Surface with Non-Uniform Heat Source/Sink," Ain Shams Eng. J. In Press, 1-8. http://dx.doi.org/10.1016/j.asej.2016.02.008

Raza, J., Rohni, A. M., and Omar, Z., 2016, "MHD Flow and Heat Transfer of $\mathrm{Cu}$-Water Nanofluid in a Semi Porous Channel with Stretching Walls," Int. J. Heat Mass Transf. 103, 336-340. http://dx.doi.org/10.1016/j.ijheatmasstransfer.2016.07.064

Hakeem, A. K. A., Saranya, S., and Ganga, B., 2017, "Comparative Study on Newtonian/Non-Newtonian Base Fluids with Magnetic/non-
Magnetic Nanoparticles over a Flat Plate with Uniform Heat Flux," J. Mol. Liq. 230, 445-452. http://dx.doi.org/10.1016/j.molliq.2016.12.087

Sulochana, C., Ashwinkumar, G. P., and Sandeep, N., 2017, "Effect of Thermophoresis and Brownian Moment on 2D MHD Nanofluid Flow over an Elongated Sheet," Defect Diffus. Forum 377, 111-126. http://dx.doi.org/10.4028/www.scientific.net/DDF.377.111

Sulochana, C., Ashwinkumar, G. P., and Sandeep, N., 2017, "Joule Heating Effect on a Continuously Moving Thin Needle in MHD Sakiadis Flow with Thermophoresis and Brownian Moment," Eur. Phys. J. PLUS 132, 387-400. http://dx.doi.org/10.1140/epjp/i2017-11633-3

Sulochana, C., Ashwinkumar, G. P., and Sandeep, N., 2017, "Boundary Layer Analysis of Persistent Moving Horizontal Needle in Magnetohydrodynamic Ferrofluid: A Numerical Study," Alexandria Eng. J. http://dx.doi.org/10.1016/j.aej.2017.08.020

Sulochana, C., Samrat, S. P., and Sandeep, N., 2017, "Boundary Layer Analysis of an Incessant Moving Needle in MHD Radiative Nanofluid with Joule Heating," Int. J. Mech. Sci. http://dx.doi.org/10.1016/j.ijmecsci.2017.05.006

Vempati, S. R., and Laxmi-Narayana-Gari, A. B., 2010, "Soret and Dufour Effects on Unsteady MHD Flow Past an Infinite Vertical Porous Plate with Thermal Radiation," Appl. Math. Mech. (English Ed. 31, 1481-1496. http://dx.doi.org/10.1007/s10483-010-1378-9

Hayat, T., Shehzad, S. A., and Alsaedi, A., 2012, "Soret and Dufour Effects on Magnetohydrodynamic (MHD) Flow of Casson Fluid," Appl. Math. Mech. 33, 1301-1312. http://dx.doi.org/10.1007/s10483-012$\underline{1623-6}$

Pal, D., and Modal, H., 2014, "Soret-Dufour Effects on Hydromagnetic Non-Darcy Convective-Radiative Heat and Mass Transfer over a Stretching Sheet in Porous Medium with Viscous Dissipation and Ohmic Heating," J. Appl. Fluid Mech. 7, 513-523.

Krishna, P. M., Sandeep, N., and Sugunamma, V., 2015, "Effects of Radiation and Chemical Reaction on MHD Convective Flow over a Permeable Stretching Surface with Suction and Heat Generation," Walailak J. Sci. Technol. 12, 831-847.

Srinivasacharya, D., Mallikarjuna, B., and Bhuvanavijaya, R., 2015, "Soret and Dufour Effects on Mixed Convection along a Vertical Wavy Surface in a Porous Medium with Variable Properties," Ain Shams Eng. J. 6, 553-564. http://dx.doi.org/10.1016/j.asej.2014.11.007

Sulochana, C., Payad, S.S., and Sandeep, N., 2016, "Non-niform Heat Source or Sink Effect on the Flow of 3D Casson Fluid in the Presence of Soret and Thermal Radiation," Int. J. Eng. Res. Africa 20, 112-129. http://dx.doi.org/10.4028/www.scientific.net/JERA.20.112

Reddy, P. S., and Chamkha, A. J., 2016, "Soret and Dufour Effects on MHD Convective Flow of $\mathrm{Al}_{2} \mathrm{O}_{3}-$ Water and $\mathrm{TiO}_{2}$-Water Nanofluids Past a Stretching Sheet in Porous Media with Heat Generation/Absorption," Adv. Powder Technol. 27, 1207-1218. http://dx.doi.org/10.1016/j.apt.2016.04.005

Sandeep, N., Sulochana, C., and Kumar, B. R., 2015, "Unsteady MHD Radiative Flow and Heat Transfer of a Dusty Nanofluid over an 
Exponentially Stretching Surface," Eng. Sci. Technol. an Int. J. http://dx.doi.org/10.1016/j.jestch.2015.06.004

Sulochana, C., and Samrat, S. P., 2017, "Unsteady MHD Radiative Flow of a Nanoliquid Past a Permeable Stretching Sheet: An Analytical Study," J. Nanofluid 6, 1-9. http://dx.doi.org/10.1166/jon.2017.1367

Rashid, M., Nadeem, S., Saleem, S., Akbar, N.S., 2017, "Flow and Heat Transfer Analysis of Jeffery Nano Fluid Impinging Obliquely over a Stretched Plate," J. Taiwan Inst. Chem. Eng. 74, 49-58. http://dx.doi.org/10.1016/j.jtice.2017.02.001

Nadeem, S., Saleem, S., 2014, "Unsteady Mixed Convection Flow of Nanofluid on a Rotating Cone with Magnetic Field," Appl. Nanosci. 4(4), 405-414. http://dx.doi.org/10.1007/s13204-013-0213-1

Babu, J., Sandeep, N., Saleem, S., 2017, "Free Convective MHD Cattaneo-Christov Flow over Three Different Geometries with Thermophoresis and Brownian Motion," Alexandria Eng. J. 56(4), 659669. http://doi.org/10.1016/j.aej.2017.01.005

Nadeem, S., Khan, A.U., Saleem, S., 2016, "A Comparative Analysis on Different Nanofluid Models for the Oscillatory Stagnation Point Flow," Eur. Phys. J. PLUS 131, 261-274. http://doi.org/10.1140/epjp/i2016$\underline{16261-9}$

Saleem, S., Nadeem, S., Awais, M., 2016, “Time Dependent Second Order Viscoelastic Fluid Flow on a Rotating Cone with Heat Generation and Chemical Reaction," J. Aerospace Eng. 29(4), 04016009.
Sulochana, C., Ashwinkumar, G.P., Sandeep, N., 2017a, "Effect of Frictional Heating on Mixed Convection Flow of Chemically Reacting Radiative Casson Nanofluid over an Inclined Porous Plate," Alexandria Eng. J. http://doi.org/10.1016/j.aej.2017.08.006

Sulochana, C., Ashwinkumar, G.P., Sandeep, N., 2017b, "Transpiration Effect on Stagnation-Point Flow of a Carreau Nanofluid in the Presence of Thermophoresis and Brownian Motion," Alexandria Eng. J. 55(2), 1151-1157. http://doi.org/10.1016/j.aej.2016.03.031

Sulochana, C., Samrat, S.P., Sandeep, N., 2017c, "Magnetohydrodynamic Radiative Nanofluid Flow over a Rotating Surface with Soret Effect," Multi. Model. Mat. Struc. http://doi.org/10.1108/MMMS-05-2017-0042

Sulochana, C., Samrat, S.P., Sandeep, N., 2018, "Numerical Investigation of Magnetohydrodynamics (MHD) Radiative Flow over a Rotating Cone in the Presence of Soret and Chemical Reaction," Propulsion and Power Research. In press. http://doi.org/10.1016/jppr.2018.01.001

Sheikholeslami, M., Rokni, H.B., 2017, "Influence of EFD Viscosity on Nanofluid Forced Convection in a Cavity with Sinusoidal Wall," J. Mol. Liq. 232, 390-395. http://doi.org/10.1016/j.molliq.2017.02.042 